\title{
Estudos de caso de aplicativos móveis no governo brasileiro
}

\author{
Alisson Wilker Andrade, Ronaldo Agra, Viviane Malheiros \\ Serpro - Serviço Federal de Processamento de Dados \\ SGAN 601 Modulo V - 70836-900 - Brasília - DF - Brasil \\ \{alisson-wilker.silva, jose-ronaldo.souza, \\ viviane.malheiros\} @serpro.gov.br
}

\begin{abstract}
In Brazil, the use of mobile internet and mobile devices, such as tablets and smartphones, has been growing in a higher pace than global mean in recent years. The use of those devices has modified the way people interact with each other and with companies around, and has also potential to reach government-government, government-company and government-citizen relationships. In this way, this paper presents challenges for building mobile applications in the context of the federal government, discussing and comparing possible existing application architectures and presenting 2 case studies built at SERPRO for the Brazilian Internal Revenue Service and for the National Department of Transport Infrastructure.
\end{abstract}

Resumo. No Brasil, o uso de internet móvel e de dispositivos móveis, como tablets e smartphones, tem crescido a taxas mais altas do que a média mundial nos últimos anos. $O$ uso desses dispositivos tem modificado a forma como as pessoas interagem entre si e com as empresas ao redor, e também tem grande potencial para alcançar as relações governo-governo, governoempresa e governo-cidadão. Nesse sentido, este artigo apresenta os desafios de construir aplicativos para dispositivos móveis no contexto do governo federal, discutindo e comparando as possíveis arquiteturas de aplicativos existentes no mercado e apresentando 2 estudos de caso de aplicativos construídos pelo SERPRO para a Receita Federal e para o DNIT.

\section{A mobilidade e sua influência no cotidiano dos brasileiros}

O uso de dispositivos móveis possibilita o acesso a serviços e informações a qualquer momento, por meio de redes sem fio e de diversos recursos, como: texto, voz, vídeo, internet, GPS, câmera, música e televisão. Estes recursos integrados podem melhorar significativamente a prestação de serviços, e, em particular, de serviços de governo eletrônico. As possibilidades vão muito além da realização de chamadas telefônicas, e são aplicáveis a diversas áreas como: educação, bancária, entretenimento, turismo e saúde [Changlin 2011].

Explorar recursos de mobilidade digital é uma oportunidade para o governo de oferecer serviços mais adequados ao cidadão, além de ter serviços governo-governo mais eficientes e dinâmicos [Nkosi 2010] [Jian 2007] [Chen Su 2010]. Em especial no Brasil, visto que a disponibilidade de acesso à Internet e o uso de dispositivos móveis cresce a taxas maiores do que a média mundial. Em 2011, houve um crescimento de quase $100 \%$ no total de acessos à Internet móvel [Jornal O Povo Online 2012]. Em maio de 2012, a difusão de smartphones já atingia 14\% da população, segundo relatório da 
Google sobre o mercado de mobilidade brasileiro [Google Inc. a 2012].

O aumento do uso de smartphones no Brasil tem impactado diretamente o cotidiano dos brasileiros: $42 \%$ dos usuários de smartphones acessam diariamente a Internet a partir de seus dispositivos móveis; $80 \%$ deles pesquisam um produto no dispositivo antes de comprar; e $31 \%$ fazem compras a partir do dispositivo [Google Inc. a 2012]. Essa nova forma de executar atividades comuns do dia a dia influencia o relacionamento de empresas com seus clientes e também tem potencial para influenciar os relacionamentos governo-governo, governo-empresa e governo-cidadão.

Nesse cenário, empresas têm adaptado seus sites e propagandas para clientes, que agora chegam a ela através de um dispositivo móvel, tanto de dentro de um ônibus, como realizando exercícios físicos num parque da cidade. Da mesma forma, para assuntos relacionados ao governo, pode-se imaginar um cidadão consultando sua certidão eleitoral ou sua matrícula na universidade por meio de um dispositivo móvel.

Além de ser uma grande oportunidade, o uso abrangente de computação móvel para governo é, também, um grande desafio. É fundamental ter infraestrutura e dominar o ambiente para explorar adequadamente questões relacionadas a particularidades dos dispositivos móveis, como: (i) limitações de memória, bateria, processamento, tamanho de tela e teclado, capacidade de armazenamento, largura de banda e consumo de energia; (ii) grande diversidade de aparelhos e plataformas de desenvolvimento (proprietárias e livres); (iii) segurança; e (iv) forma de publicação de aplicativos [Al Thunibat 2010].

Em 2012, o Serpro desenvolveu e publicou aplicações móveis para a Receita Federal do Brasil [Apple Inc. a 2013] [Google Inc. b 2013] [Apple Inc. b 2013] [Google Inc. c 2013] e para o DNIT (Departamento Nacional de Infraestrutura de Transportes) [DNIT a 2012] [DNIT b 2012]. O desenvolvimento destas soluções suscitou o estudo das características do desenvolvimento para dispositivos móveis e discussões arquiteturais abrangentes. A experiência obtida com estes projetos está sendo utilizada na definição de ações e modelos corporativos para habilitar o Serpro a oferecer soluções inovadoras para o governo [Andrade et al. 2012].

Neste contexto, este artigo apresenta: (i) possíveis arquiteturas de aplicações para dispositivos móveis e como escolher a mais adequada a partir de seus requisitos; e (ii) dois estudos de caso de mobilidade para governo desenvolvidos pelo Serpro.

\section{Arquiteturas de desenvolvimento para dispositivos móveis}

O desenvolvimento para dispositivos móveis engloba dois cenários: (i) adaptação de sítios e portais web para utilização a partir de qualquer tablet ou smartphone; e (ii) desenvolvimento de aplicações nativas adequadas para cada plataforma. Cada um desses cenários requer uma arquitetura de solução específica, que valorize suas características principais. Assim, esta seção apresenta três possíveis arquiteturas: (i) uma arquitetura para desenvolvimento de conteúdo web móvel, (ii) outra para desenvolvimento de conteúdo nativo móvel, (iii) e uma terceira arquitetura que é chamada híbrida, pois engloba tanto o desenvolvimento nativo como o desenvolvimento web móvel, buscando valorizar o que há de melhor em cada cenário.

\subsection{Arquitetura para desenvolvimento de conteúdo web móvel}

O desenvolvimento de aplicações para dispositivos móveis engloba a construção de 
conteúdo web adaptado para a interação via estes dispositivos (Figura 1). Nesse caso, o usuário acessa um sítio ou aplicação web através do navegador web instalado em seu smartphone ou tablet. Através de uma conexão com a Internet ou a intranet, o navegador realiza uma requisição ao servidor web que contém o conteúdo web da aplicação ou sítio. Ao receber a requisição, este identifica qual é o dispositivo que está solicitando o conteúdo web e realiza o redirecionamento para a página correta, seja um dispositivo móvel ou um computador pessoal. Por exemplo, o navegador desktop pode acessar o sítio http://www.serpro.gov.br enquanto o navegador móvel acessa http://m.serpro.gov.br, mesmo que ambos tenham feito uma requisição por http://www.serpro.gov.br.

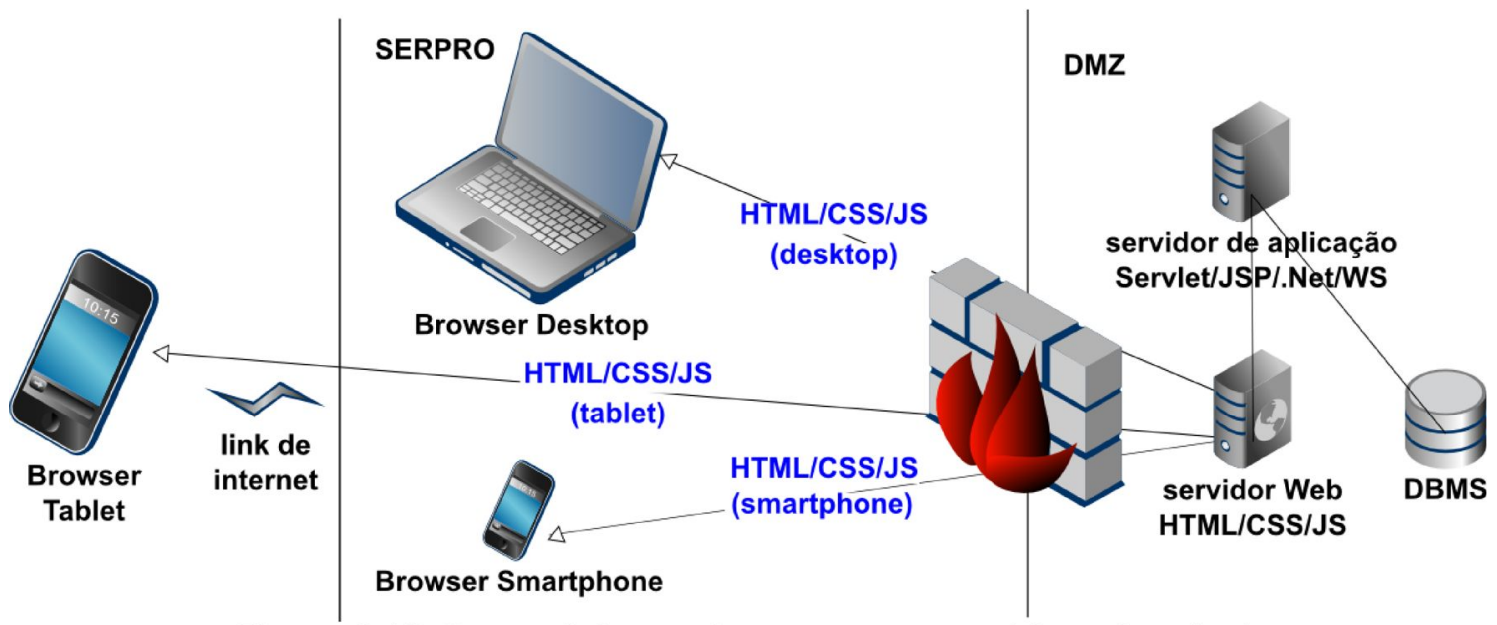

Figura 1: Visão geral de arquitetura para conteúdo web móvel.

Outra maneira de instanciar esta arquitetura é possuir apenas um conteúdo ou aplicação web e usar layouts diferentes para cada tipo de dispositivo, o que é chamado de design responsivo (responsive design). Design Responsivo é uma técnica de estruturação de HTML e CSS na qual a página adequa-se ao navegador do usuário sem a necessidade de criar vários conteúdos para cada resolução de tela. Nesse caso, o CSS pode utilizar o recurso de "media query" para identificar o tamanho de tela do navegador e adaptar o layout do conteúdo ou aplicação web para um tamanho específico.

A melhor escolha entre essas alternativas deve ser guiada pelo tipo de conteúdo web, os objetivos de sua publicação e o perfil dos usuários esperados. Porém, em ambos os casos, é importante ressaltar que a aplicação ou o conteúdo web desenvolvido para tablet pode ser bastante diferente daquele desenvolvido com foco em smartphones. Isto porque as dimensões e a resolução de tela, bem como a forma de uso, são bem distintas entre esses dispositivos. Assim, normalmente, o conteúdo servido para smartphones é bem mais conciso, principalmente se comparado ao conteúdo servido para um navegador desktop [Nielsen 2012].

\subsection{Arquitetura para aplicação nativa móvel}

Outra possibilidade de desenvolvimento para dispositivos móveis é a construção de aplicações nativas para cada plataforma móvel, como Android, iOS, Blackberry ou Windows Phone (Figura 2). Nesse caso, o desenvolvedor utiliza o ambiente de desenvolvimento e as bibliotecas fornecidas pelo fabricante da plataforma para desenvolver a aplicação. 


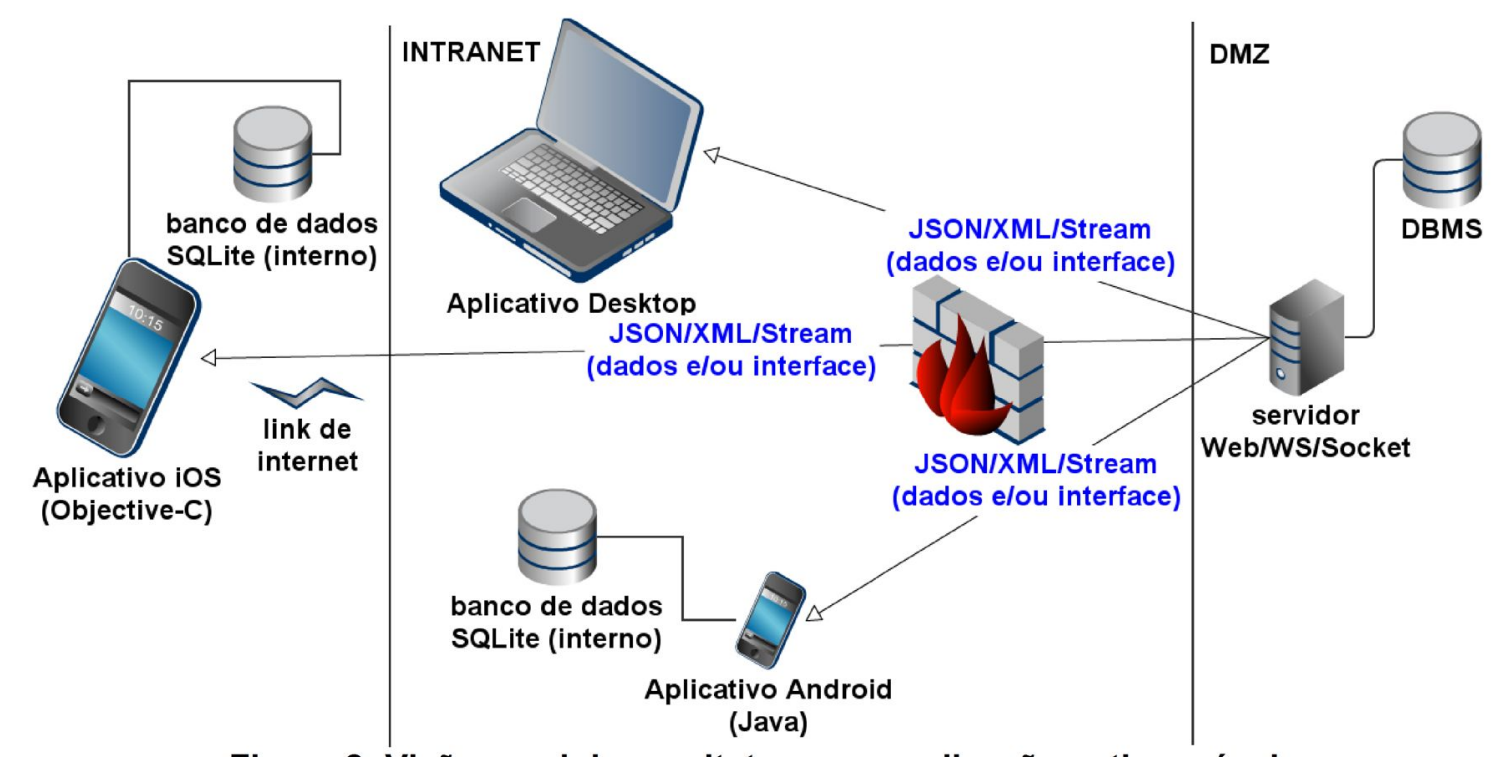

Figura 2: Visão geral de arquitetura para aplicação nativa móvel.

A aplicação nativa normalmente tem um visual mais integrado com a plataforma do dispositivo, visto que os componentes visuais como botões, menus e listas, por exemplo, são fornecidos e estilizados pela própria plataforma. Além disso, nesta arquitetura de desenvolvimento, é possível utilizar diversos recursos de hardware do dispositivo, como acelerômetro, GPS, bússola e câmera, que normalmente não podem ser acessados diretamente a partir de uma aplicação web executada em um navegador de Internet. Essa possibilidade leva à criação de aplicações muito mais ricas em termos de funcionalidades e integração com o dispositivo móvel.

Para o armazenamento de informações, uma aplicação nativa pode realizar o armazenamento local tanto em arquivos como em banco de dados SQLite. O SQLite é um banco de dados transacional leve e multiplataforma utilizado pelas principais plataformas móveis, como Android e iPhone [SQLite 2012]. Ele permite o armazenamento de textos, números e dados binários e pode ser consultado através da linguagem SQL diretamente da aplicação ou via linha de comando.

Normalmente, uma aplicação desenvolvida nativamente será submetida para validação e publicação em uma store ${ }^{l}$ de aplicações a partir da qual o usuário poderá instalá-la em seu dispositivo móvel e, em seguida, acessá-la através de um ícone no menu de aplicações ou na tela principal do aparelho. Assim, o fabricante da plataforma se responsabiliza por toda a infraestrutura para publicação e distribuição das aplicações. Desta forma, o desenvolvedor pode focar seus esforços do desenvolvimento da aplicação em si, não tendo que se preocupar com a forma pela qual seu usuário irá adquirir o software.

\subsection{Arquitetura para aplicação nativa + conteúdo web móvel}

A terceira abordagem para desenvolvimento de conteúdo para dispositivos móveis é a composição de conteúdo nativo com conteúdo web, também conhecida como aplicação híbrida. Uma aplicação híbrida normalmente é construída quase completamente utilizando-se de linguagens padronizadas como HTML, CSS e JavaScript, e apenas uma

1 Store ou loja de aplicativos é um catálogo de aplicações que todas as plataformas utilizam para disponibilizar aos usuários os aplicativos existentes para a plataforma. 
pequena parte em linguagem nativa da plataforma. Dessa forma, boa parte do código de uma aplicação híbrida construída para Android, por exemplo, pode ser reaproveitada no desenvolvimento para outras plataformas.

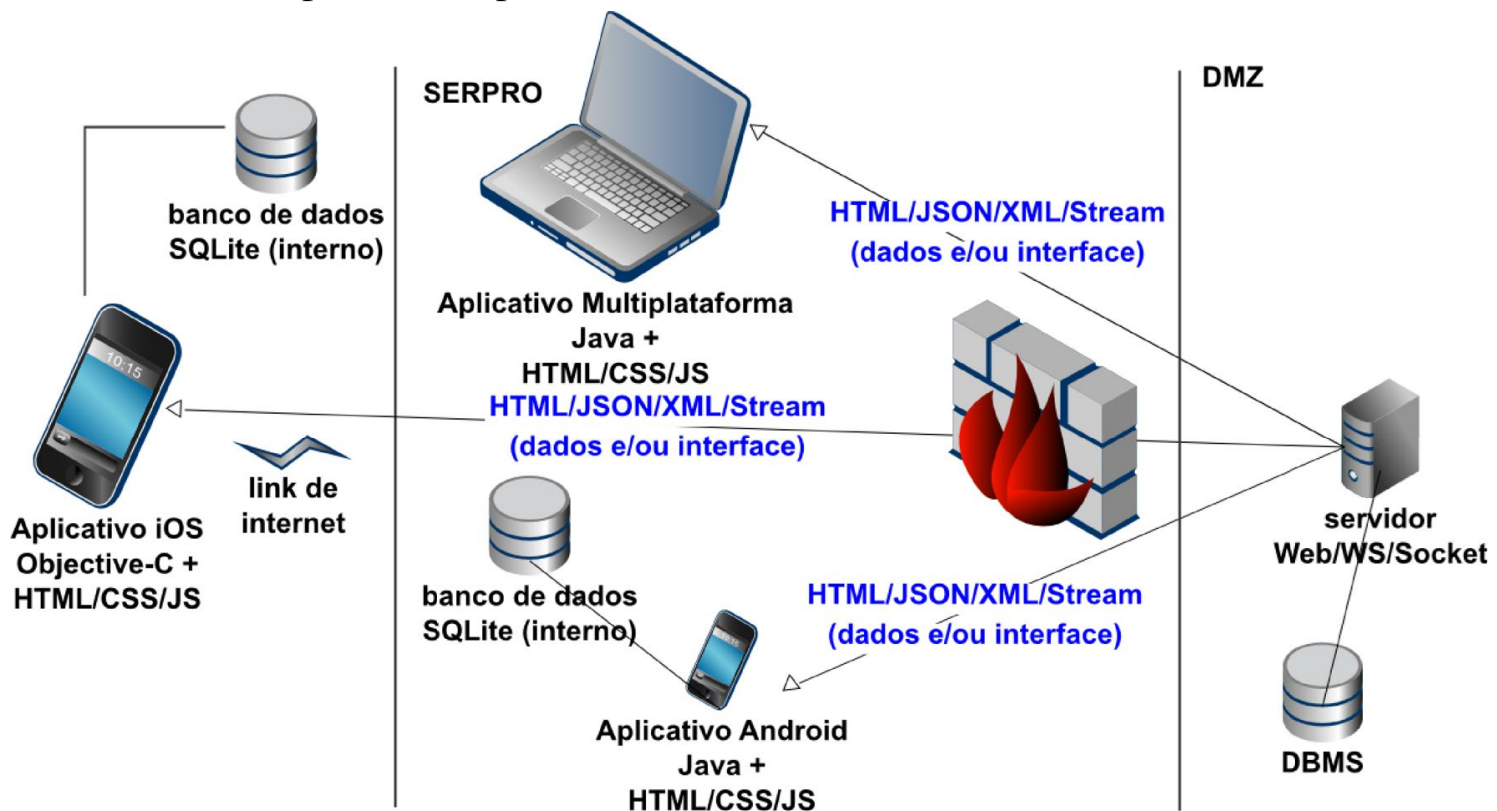

Figura 3: Visão geral de arquitetura para aplicação nativa + conteúdo web móvel.

Conforme Figura 3, a aplicação híbrida pode se comunicar com um servidor remoto através de um link de Internet ou com um servidor local através de uma rede WiFi na intranet da organização. As informações trafegadas entre aplicação móvel e servidor podem ser dados ou descrições de componentes de interface a serem renderizados pela aplicação, assim como pode ser feito em aplicações nativas. Dessa forma, quando uma modificação afeta apenas a interface de usuário, essa alteração pode ser feita apenas no servidor remoto, evitando-se um novo ciclo de validação e publicação na store de aplicações.

A aplicação híbrida também permite tanto o armazenamento local em arquivos como o armazenamento em banco de dados SQLite. Normalmente, o acesso ao banco de dados SQLite é escrito na linguagem nativa da plataforma alvo. Porém, alguns frameworks de desenvolvimento de aplicações móveis híbridas oferecem API específica para que este acesso seja escrito em JavaScript [Adobe Systems 2012].

Aplicações que obedecem a essa arquitetura são instaladas no dispositivo móvel a partir de uma store de aplicativos. Portanto, também estão sujeitas ao ciclo de validação e publicação determinado por cada store. Além disso, assim como uma aplicação nativa, as aplicações híbridas são acessadas via um ícone da aplicação na tela do dispositivo e podem usar recursos do dispositivo, como acelerômetro, GPS, bússola e câmera.

\subsection{Orientações para escolha da arquitetura}

Conforme apresentado na Tabela 1, cada arquitetura possui vantagens e desvantagens que devem ser consideradas durante a construção de qualquer aplicação móvel ${ }^{2}$. A decisão por qual utilizar deve ser tomada pela equipe do projeto e deve levar em

2 O conteúdo da Tabela 1 é uma compilação de informações encontradas nas várias referências consultadas e também um resultado da experiência do SERPRO no assunto. 
consideração a análise de alguns critérios tecnológicos, além dos requisitos da aplicação a ser construída.

Dentre os critérios a serem avaliados no momento de escolha da arquitetura, pode-se citar: (i) o acesso aos recursos de hardware do dispositivo móvel, (ii) o armazenamento local de informações, (iii) a restrição de conectividade com a Internet/intranet e (iv) o visual integrado à plataforma onde será executada. A Figura 4 apresenta um fluxo simples de tomada de decisão contendo os principais critérios a serem observados para a escolha da melhor arquitetura de desenvolvimento de um aplicativo para dispositivos móveis.

Desta forma, caso seja imprescindível o acesso aos recursos de hardware do dispositivo e a conectividade com a Internet seja restrita, as arquiteturas que envolvam código nativo devem ser priorizadas. Por outro lado, se não existem essas restrições e

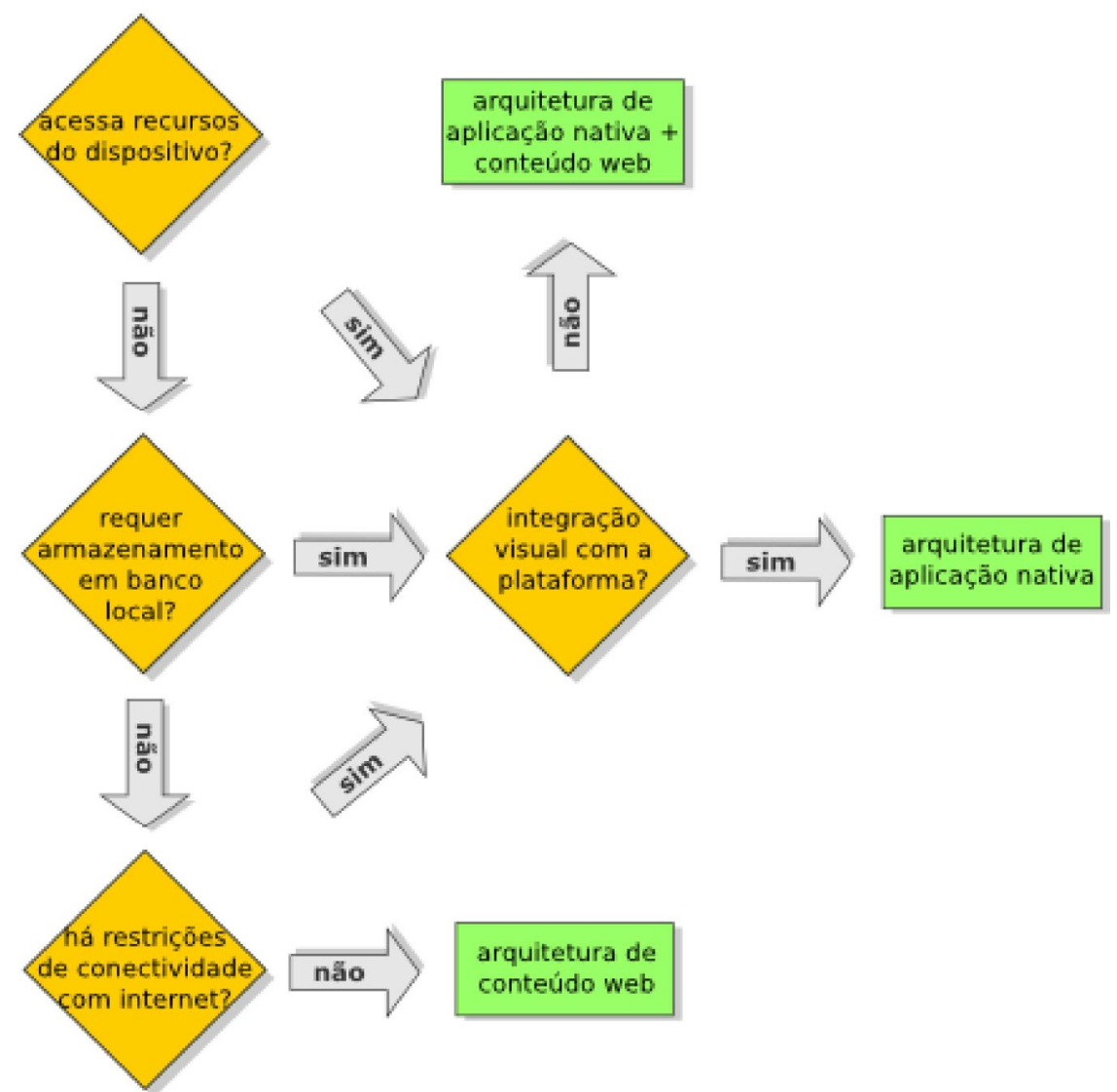

Figura 4: Fluxo de decisão para escolha da arquitetura. 
Tabela 1: Quadro comparativo entre as arquiteturas para o desenvolvimento de aplicações móveis.

\begin{tabular}{|c|c|c|}
\hline Arquiteturas & Vantagens & Desvantagens \\
\hline $\begin{array}{l}\text { Arquitetura para desenvolvimento } \\
\text { de conteúdo web móvel }\end{array}$ & $\begin{array}{l}\text { Utilização de um único endereço (URL) para acessar a aplicação; } \\
\text { Facilidade de implantar novas versões da aplicação; } \\
\text { Maior segurança para regras de negócio; } \\
\text { Reaproveitamento de código da interface (HTML, CSS e JavaScript) e } \\
\text { da lógica de negócio (hospedada em um servidor); }\end{array}$ & $\begin{array}{l}\text { Dependência de conectividade com a Internet; } \\
\text { Possibilidade de falha ou restrição de desempenho na aplicação devido } \\
\text { ao navegador web escolhido pelo usuário; } \\
\text { Necessidade de testes criteriosos em diversas versões de navegadores } \\
\text { antes da liberação; } \\
\text { Limitação de acesso aos recursos de hardware do dispositivo móvel } \\
\text { (ex.: câmera, banco de dados local). }\end{array}$ \\
\hline $\begin{array}{l}\text { Arquitetura para aplicação nativa } \\
\text { móvel }\end{array}$ & $\begin{array}{l}\text { Integração da aplicação com o visual da plataforma em que ela é } \\
\text { executada; } \\
\text { Integração da aplicação com os recursos do dispositivo móvel, como } \\
\text { acelerômetro, GPS, câmera e banco de dados local, por exemplo; } \\
\text { Monitoração das estatísticas de download e de satisfação dos usuários } \\
\text { em relação a uma aplicação baixada da store de aplicações; } \\
\text { Mais possibilidades de protocolos de comunicação com o servidor } \\
\text { remoto, além daqueles suportados pelos navegadores web. }\end{array}$ & $\begin{array}{l}\text { A migração dos usuários para uma nova versão da aplicação não é } \\
\text { controlada; } \\
\text { Existência de um custo extra de publicação da aplicação na store de } \\
\text { aplicações do fabricante; } \\
\text { Especialização da equipe de desenvolvimento em uma ou mais } \\
\text { plataformas móveis; } \\
\text { Necessidade de disponibilizar o código-fonte da aplicação para o } \\
\text { proprietário da store de aplicações durante o processo de validação e } \\
\text { publicação. }\end{array}$ \\
\hline $\begin{array}{l}\text { Arquitetura para aplicação nativa } \\
+ \text { conteúdo web móvel }\end{array}$ & $\begin{array}{l}\text { Integração da aplicação com os recursos do dispositivo móvel, como } \\
\text { acelerômetro, GPS, câmera e banco de dados local, por exemplo; } \\
\text { Escrita da maior parte do código em linguagem padrão multiplataforma } \\
\text { (HTML, CSS e JavaScript), possibilitando o reaproveitamento de } \\
\text { código; } \\
\text { Possibilidade de redução da quantidade de desenvolvedores } \\
\text { especializados em cada plataforma; } \\
\text { Monitoração das estatísticas de download e de satisfação dos usuários } \\
\text { em relação a uma aplicação baixada da store de aplicações; } \\
\text { Mais possibilidades de protocolos de comunicação com o servidor } \\
\text { remoto, além daqueles suportados pelos navegadores web. }\end{array}$ & $\begin{array}{l}\text { Demora para validar e publicar a aplicação em uma store de aplicações; } \\
\text { Maior esforço para customizar os componentes da aplicação para } \\
\text { deixar sua aparência e comportamento similares ao da plataforma; } \\
\text { A migração dos usuários para uma nova versão da aplicação não é } \\
\text { controlada; } \\
\text { Existência de um custo extra de publicação da aplicação na store de } \\
\text { aplicações do fabricante; } \\
\text { Necessidade de disponibilizar o código da aplicação para o proprietário } \\
\text { da store de aplicações durante o processo de validação e publicação. }\end{array}$ \\
\hline
\end{tabular}


tampouco a necessidade de integração visual da aplicação com a plataforma, a arquitetura de conteúdo web pode ser a mais indicada. Esses são apenas dois exemplos de análise sobre os critérios apresentados acima, outras combinações podem ser realizadas de forma a subsidiar a decisão da equipe de desenvolvimento e arquitetura do projeto.

Independentemente de todos os critérios apresentados, pode ser interessante avaliar também as habilidades e a quantidade de pessoas na equipe que irá desenvolver a aplicação. A arquitetura nativa pode requerer mais desenvolvedores especializados em cada plataforma móvel (p. ex.: Android e iOS), enquanto as arquiteturas web e híbrida podem requerer menos pessoal especializado, visto que boa parte do código pode ser desenvolvido em linguagem multiplataforma, como HTML, CSS e JavaScript.

\section{Aplicações móveis}

As arquiteturas apresentadas foram experimentadas em protótipos. Os protótipos desenvolvidos demonstraram que, para os casos estudados, a arquitetura para desenvolvimento de aplicações nativas se mostrou mais vantajosa. O Serpro desenvolveu e publicou aplicações móveis nativas para a RFB (Receita Federal do Brasil) e para o DNIT (Departamento Nacional de Infraestrutura de Transportes). Em junho de 2012, a RFB disponibilizou para os contribuintes o aplicativo móvel Pessoa Física e, desde julho, o SIESC Mobile está disponível para apoiar o DNIT na fiscalização de obras públicas em andamento. As subseções a seguir apresentam as características dessas aplicações e analisam a seleção da arquitetura mais adequada para cada solução.

\subsection{Aplicação Pessoa Física (RFB)}

A aplicação Pessoa Física (Figura 5.a), disponibilizada pela RFB, permite que um contribuinte consulte a situação de sua restituição de Imposto de Renda (Figura 5.b). Além disso, um cidadão pode consultar a situação do seu Cadastro de Pessoa Física $(\mathrm{CPF})$, identificando se está regular ou se existe alguma pendência. A aplicação também apresenta informações gerais sobre o Imposto de Renda e o processo de restituição.

O aplicativo Pessoa Física foi lançado no dia 06 de junho de 2012 e tem recebido avaliações muito positivas, desde então. $\mathrm{Na}$ Google $\mathrm{Play}^{3}$, a loja de aplicativos Android, a aplicação está avaliada com 4,6 estrelas, de um máximo de 5 estrelas [Google Inc. b 2013] (Figura 5.c). Já na App Store ${ }^{4}$, a loja de aplicativos iOS, a aplicação foi avaliada por 85 usuários, com nota 4 de um total de 5 [Apple Inc. a 2013] (Figura 5.d).

A solução foi construída utilizando-se de linguagens e tecnologias disponíveis para cada plataforma específica, ou seja, um exemplo de uso da arquitetura de aplicações nativas. A decisão pelo desenvolvimento com a arquitetura nativa foi baseada nos seguintes requisitos: (i) necessidade de integração visual com cada plataforma; (ii) necessidade de disponibilizar nas lojas de aplicativos para dispositivos móveis para facilitar a descoberta dos aplicativos; (iii) necessidade de adequar as imagens por

3 Google Play é a "loja" de aplicativos Android. Esta "loja", além de disponibilizar o aplicativo, permite que o usuário o avalie com uma nota até 5 .

4 App Store é a "loja" de aplicativos da Apple. Esta "loja", além de disponibilizar o aplicativo, permite que o usuário o avalie com uma nota até 5. 
plataforma para seguir o padrão de qualidade visual da RFB.

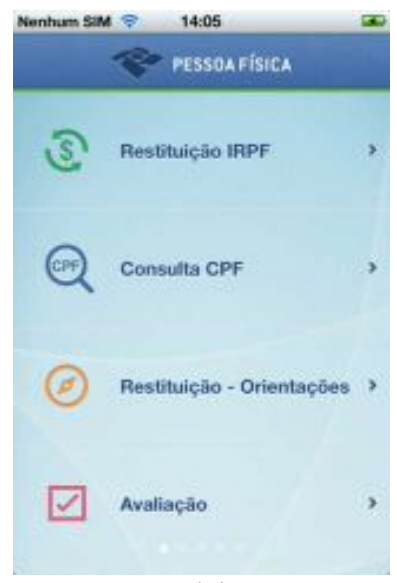

(a)

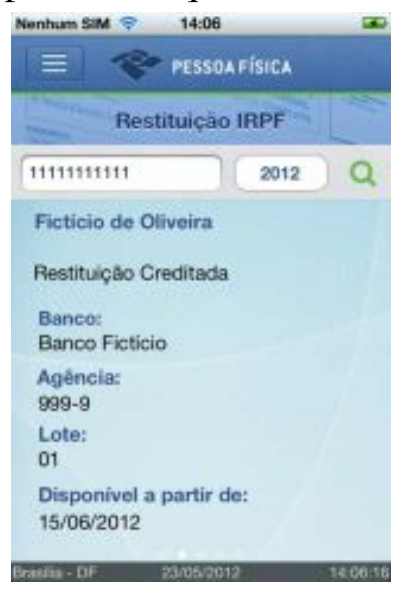

(b)

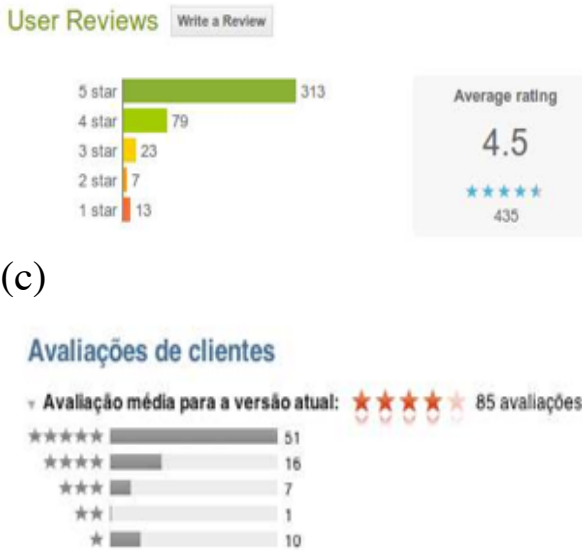

(d)

Figura 5: Aplicativo Pessoa Física: (a) Tela principal; (b) consulta da restituição do IRPF [Apple Inc. a 2013]; avaliação do aplicativo (c) na loja Google Play [Google Inc. b 2013] e (d) na loja App Store [Apple Inc. a 2013]

A decisão se mostrou acertada, entretanto a escolha da arquitetura nativa requereu o desenvolvimento de dois aplicativos: um para a plataforma Android e outro para iOS. Com isso, duas equipes foram mobilizadas e, após a fase de requisitos, as fases do desenvolvimento foram replicadas. Além do retrabalho, essa estratégia demandou sincronização constante entre as equipes para garantir a proximidade entre as duas versões. A complexidade do desenvolvimento e manutenção aumenta à medida que novas plataformas são consideradas. Assim, esta deve ser uma preocupação ao optar-se pelo desenvolvimento nativo. Uma outra questão vivenciada foi que a publicação nas lojas pode impactar no cronograma de disponibilização dos aplicativos. A publicação, ou não, de um aplicativo depende de uma avaliação de cada loja, e não há como priorizar publicações. Assim, uma avaliação para publicação pode demorar horas ou dias, dependendo da loja para a qual se está submetendo o aplicativo.

\subsection{Aplicação SIESC Mobile}

Outro aplicativo móvel desenvolvido pelo SERPRO foi o SIESC Mobile (Figura 6). Seu objetivo principal é melhorar a qualidade das fiscalizações das obras que são de responsabilidade do DNIT, proporcionando também redução de tempo e custo. Antes do SIESC Mobile, um engenheiro (que poderia ser de uma empreiteira contratada pelo governo) chegava à obra com um formulário impresso para preencher todas as características da obra (localização, altitude, comprimento, tipo...) e sua situação atual (falhas na estrutura, avanço da construção em relação à última medição...). Após o preenchimento desse formulário, as informações em papel eram transcritas para o Sistema de Acompanhamento de Contratos (SIAC) ${ }^{5}$.

5 https://sisdnit.dnit.gov.br/ 


\section{OSIESCH}
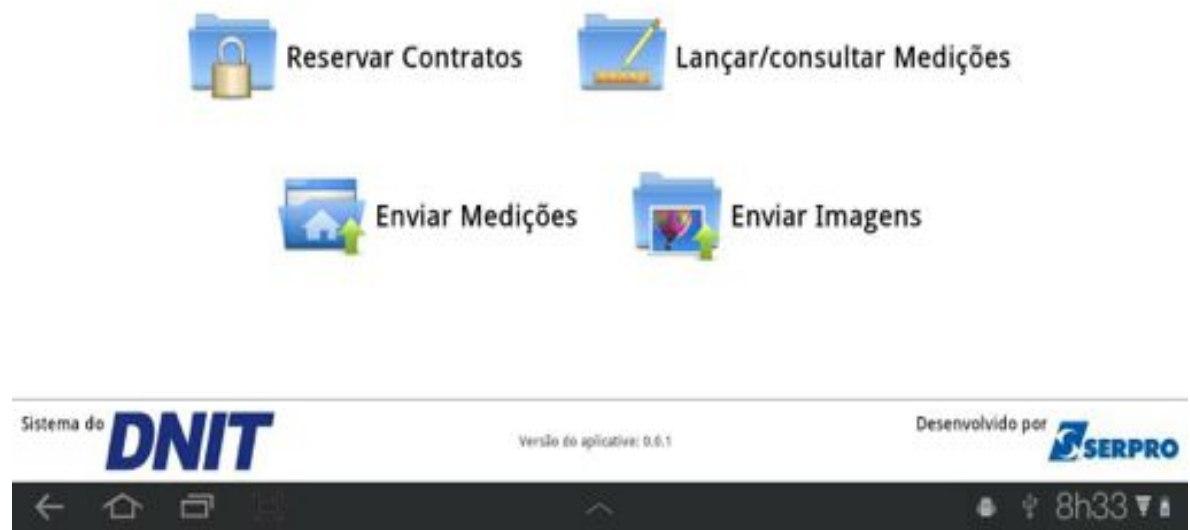

Figura 6: Utilização do SIESC Mobile para efetuar uma medição de contrato.

A partir do SIESC Mobile, as informações são captadas in loco. Recursos integrados, como GPS e câmera, permitem a complementação das informações com dados precisos de localização e fotos do andamento da obra, que mostram melhor a situação atual da mesma. Além disso, várias validações na entrada dos dados aumentam a qualidade das informações, agilizam o processo e evitam retornos desnecessários à obra para correção de dados inválidos, que algumas vezes só eram identificados no escritório.

A expectativa é que a solução SIESC Mobile possibilite quitar os serviços contratados com maior eficiência e que o uso da ferramenta traga maior agilidade em comparação ao processo anterior, baseado em formulários de papel, e também que os valores dos contratos possam ser reduzidos mediante a redução dos custos de fiscalização para a empresa contratada.

Assim como o Pessoa Física, o SIESC Mobile é um exemplo de aplicação construída usando a arquitetura nativa, ou seja, utilizando-se de tecnologias que executam apenas em uma plataforma específica. A decisão pelo desenvolvimento nativo foi baseada nos seguintes requisitos: (i) necessidade de utilizar recursos específicos dos dispositivos, no caso, câmera e celular; (ii) restrições de conectividade com a internet, já que as obras estão espalhadas pelo Brasil, muitas vezes, longe de centros urbanos, como é o caso de estradas; e (iii) necessidade de armazenamento local, já que centenas de informações são capturadas pelo dispositivo offline.

No caso do SIESC, a plataforma escolhida foi o Android. Essa escolha foi baseada no fato de que esta é uma plataforma aberta e que possui dispositivos a preços mais acessíveis. Essa decisão foi possível porque, diferentemente da aplicação Pessoa Física, que foi desenvolvida para uso pelo cidadão, o SIESC Mobile foi construído para ser utilizado por engenheiros do DNIT e de empresas terceirizadas que fiscalizam obras de construção civil governamentais.

O aplicativo não foi disponibilizado na loja de aplicativos Google Play, já que seu público-alvo é específico. Ao invés disso, a aplicação foi disponibilizada no próprio 
portal do DNIT, através de um link para download com instruções de instalação e uso. A distribuição de aplicativos por meio deste portal já é natural para o público-alvo do aplicativo.

\section{Conclusão}

Este artigo apresentou um consolidado de dados que mostram que a presença da mobilidade digital no Brasil tem crescido a cada ano e que, muitas vezes, o ritmo desse crescimento tem sido maior do que o observado em outros países. A presença da mobilidade no Brasil se evidencia através do crescimento do uso da Internet móvel e do aumento significativo de vendas de dispositivos móveis a cada ano.

Esse crescimento tem impacto significativo na vida dos brasileiros, principalmente pelo uso frequente de aplicativos que utilizam recursos embarcados nos dispositivos móveis, como: GPS, câmera, acelerômetro, entre outros. Esses aplicativos são utilizados principalmente para comunicação, informação e entretenimento, e podem ser construídos para plataformas específicas, como Android e iOS, ou como aplicação $W e b$ para acesso via navegadores web móveis. Assim, diferentes tipos de arquiteturas para aplicações móveis foram discutidos neste artigo, apresentando suas vantagens e desvantagens com base em requisitos e contexto de uso das soluções ${ }^{6}$.

No âmbito do governo federal brasileiro, foram apresentados dois estudos de caso desenvolvidos pelo SERPRO, para os clientes Receita Federal do Brasil e DNIT. Esses estudos de caso mostraram exemplos de como a mobilidade digital pode ser utilizada para prover serviços de governo para o cidadão, como é o caso do aplicativo Pessoa Física, bem com para melhorar a eficiência das atividades de fiscalização executadas pelo Estado, como é o caso do aplicativo SIESC Mobile.

No cenário internacional, outros países também têm investido em mobilidade para serviços de governo. Na Estônia, por exemplo, dispositivos móveis são usados em sistemas de estacionamento e para melhorar a comunicação entre casa e escola e, em Bangladesh, o governo envia mensagens de texto para alertar os cidadãos sobre desastres naturais. Isso mostra que o Brasil não está sozinho e que ainda há muito a evoluir nesse tema.

Por isso, apesar do crescimento já evidenciado, a tendência da mobilidade no Brasil ainda é de crescimento. Nesse sentido, o SERPRO tem investido fortemente em preparar-se e aproveitar a oportunidade de prover soluções móveis para o governo e para o cidadão melhorando a qualidade dos serviços prestados e possibilitando uma aproximação maior dos serviços de governo com a população. Em especial, a Copa do Mundo 2014 e as Olimpíadas 2016 serão ótimas oportunidades para disponibilizar uma variedade grande de serviços móveis, que podem ajudar as pessoas que irão participar desses eventos.

\section{Referências}

Al Thunibat, A. (2010) Mobile government services in Malaysia: Challenges and opportunities. In Proceedings of 2010 International Symposium in Information Technology (ITSim), Volume 3, p. 1244-1249.

Adobe Systems
"PhoneGap Storage",

6 Mais detalhes sobre os estudos de caso e arquiteturas apresentados neste artigo podem ser encontrados em [Andrade et al. 2012]. 
http://docs.phonegap.com/en/1.2.0/phonegap storage storage.md.html, Outubro.

Andrade, A. W., Agra, R. e Malheiros, V. (2012) "Mobilidade digital aplicada ao governo brasileiro", $\quad$ http://funag.gov.br/biblioteca/index.php? option=com_docman\&task=doc_download\&gid=602\&Itemid=41, Dezembro.

Apple Inc. a (2013) "Pessoa Física", https://itunes.apple.com/br/app/pessoafisica/id529883041?mt=8, Janeiro.

Apple Inc. b (2013) "Viajantes no Exterior", https://itunes.apple.com/br/app/viajantesno-exterior/id547724184? mt=8, Janeiro.

Changlin Wang. (2011) M-Government Use: Technology, Context and Environment Determinants. In Computer Engineering and Management Sciences (ICM), 2011 International Conference on Information Technology, Volume 3, p. 290-293.

Chen Su. (2010) A General Review of Mobile E-Government in China. In Proceedings of 2010 International Conference on Multimedia Information Networking and Security (MINES), p. 733-737.

DNIT a (2012) "SIESC Mobile", https://gestao.dnit.gov.br/sistemas-gerenciais/siesc, Outubro.

DNIT b (2012) "SGO Mobile", http://www.dnit.gov.br/sistemas-gerenciais/sgo, Outubro.

Google Inc. a (2012) "Nosso Planeta Mobile: Brasil", http://services.google.com/fh/files/blogs/our mobile planet brazil pt BR.pdf, Outubro.

Google Inc. b (2013) "Pessoa Física", https://play.google.com/store/apps/details? id=br.gov.fazenda.receita.pessoafisica, Janeiro.

Google Inc. c c (2013) "Viajantes no Exterior", https://play.google.com/store/apps/details?

id=br.gov.fazenda.receita.viajantesexterior, Janeiro.

Infraero (2012) "Voos Online”, http://www.infraero.gov.br/fiquepordentro/\#/download, Outubro.

Jian Tong Cao. (2007) Application of M-government system in Beijing Municipal Government. In Proceedings of IEEE International Conference on Systems, Man and Cybernetics, 2007 (ISIC), p. 3220-3224.

Jornal O Povo Online (2012) “Acesso a Internet móvel no Brasil praticamente dobra", http://www.opovo.com.br/app/opovo/tendencias/2012/01/27/noticiasjornaltendencias ,2774145/acesso-a-internet-movel-no-brasil-praticamente-dobra.shtml, Outubro.

Nielsen, J. (2012) "Mobile Content: If in Doubt, Leave It Out", http://www.useit.com/alertbox/mobile-writing.html, Outubro.

Nkosi, M. (2010) Mobile government for improved public service provision in South Africa. In Proceedings of IST-Africa 2010, p. 1-8.

SQLite (2012) "SQLite”, http://www.sqlite.org/features.html, Outubro. 